Case Report

\title{
A Novel Technique for Laparoscopic Salvage of CAPD Catheter Malfunction and Migration: The Santosh-PGI Hanging Loop Technique
}

\author{
Santosh Kumar, ${ }^{1}$ Shivanshu Singh, ${ }^{1}$ Aditya Prakash Sharma, ${ }^{1}$ and Manish Rathi ${ }^{2}$ \\ ${ }^{1}$ Department of Urology, Postgraduate Institute of Medical Education and Research, Chandigarh 160012, India \\ ${ }^{2}$ Department of Nephrology, Postgraduate Institute of Medical Education and Research, \\ Chandigarh 160012, India
}

Correspondence should be addressed to Santosh Kumar; santoshsp1967jaimatadi@yahoo.co.in

Received 30 December 2014; Revised 13 March 2015; Accepted 16 March 2015

Academic Editor: Kostas C. Siamopoulos

Copyright (C) 2015 Santosh Kumar et al. This is an open access article distributed under the Creative Commons Attribution License, which permits unrestricted use, distribution, and reproduction in any medium, provided the original work is properly cited.

CAPD catheter malfunction is a common problem. Obstruction due to wrapping by appendices epiploicae of sigmoid colon has been rarely reported in literature. We report a case of CAPD catheter malfunction caused by catheter tip migration and obstruction by appendices epiploicae that was successfully managed by laparoscopic hanging loop technique. This case report highlights the ease with which epiplopexy can be performed and catheter tip migration can be prevented by this innovative laparoscopic procedure.

\section{Introduction}

CAPD (Continuous Ambulatory Peritoneal Dialysis) is a prevalent mode of renal replacement therapy with nearly 197,000 ESRD patients using it globally [1]. One of the important aspects of CAPD catheter is its adequate function defined as one that allows adequate inflow and/or outflow of dialysate solution [2]. CAPD catheter malfunction is one of the major causes for its discontinuation. Catheter malfunction can result from its luminal occlusion due to omental or small bowel wrapping, malposition, or migration of catheter. Obstruction due to appendices epiploicae of the sigmoid colon is a rarely reported etiology [3]. Laparoscopic salvage of malfunctioning catheters helps in reducing patient's morbidity and need of intermediary hemodialysis. However, recurrent malfunction had motivated surgeons to innovate newer methods of laparoscopic CAPD catheter salvage. We report a case of CAPD catheter occlusion caused by wrapping of appendices epiploicae of sigmoid colon, a rare cause. Laparoscopic "Santosh-PGI Hanging Loop Technique," an innovative concept with multiple benefits, salvaged it.

\section{Case Report}

A 54-year-old gentleman having diabetes and hypertension was diagnosed as end-stage renal disease requiring renal replacement therapy. He opted for CAPD. His serum creatinine was $7.9 \mathrm{mg} / \mathrm{dL}$. Straight Tenckhoff CAPD catheter insertion was done by standard open technique. Patient was discharged on postoperative day three. Two weeks after insertion, CAPD initiation was planned. However, there was slow inflow and a trickling outflow. Diagnosis of CAPD catheter malfunction was made. X-ray abdomen revealed malposition of CAPD catheter (Figure 1(a)). It was lying outside the pelvis in left lateral abdomen. Initial management with laxatives, bowel enema, failed with no improvement in dialysate flux. Repositioning of CAPD catheter was attempted under fluoroscopic guidance but it was unsuccessful. Keeping a diagnosis of CAPD catheter malposition with suspected omental wrapping, decision was made for diagnostic laparoscopy along with a salvage procedure as required.

Under general anesthesia, laparoscopy was performed with the patient in supine position with Trendelenburg tilt. Three ports were placed including one $12 \mathrm{~mm}$ camera and 


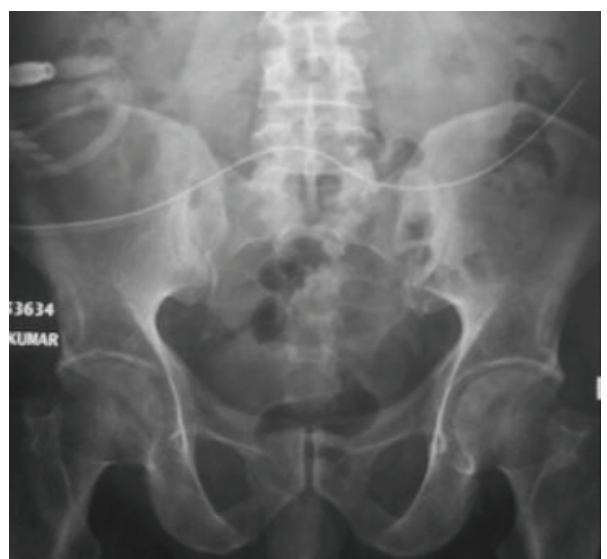

(a)

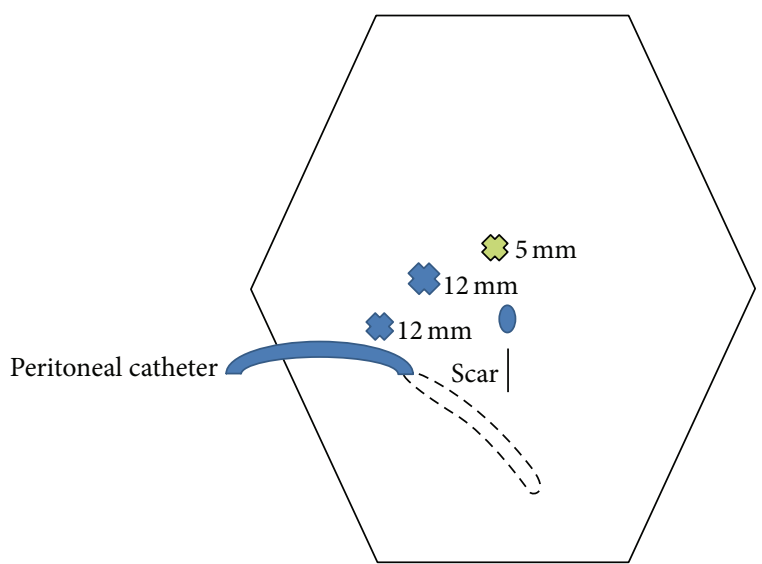

(b)

FIGURE 1: X-ray abdomen showing migrated CAPD catheter lying outside the pelvis (a). Illustration of laparoscopic port placement (b). The middle $12 \mathrm{~mm}$ blue cross is the camera port while the corners $12 \mathrm{~mm}$ and $5 \mathrm{~mm}$ are the working ports.

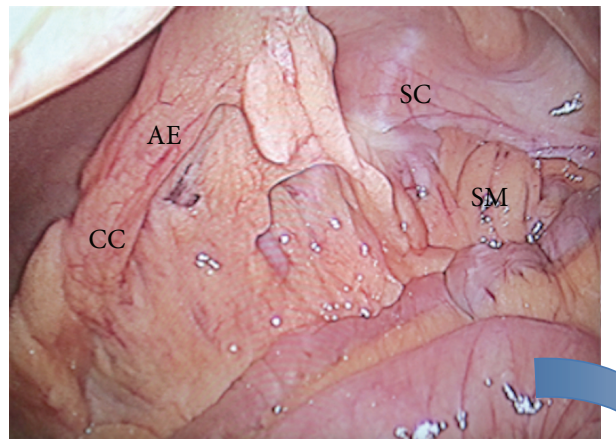

(a)

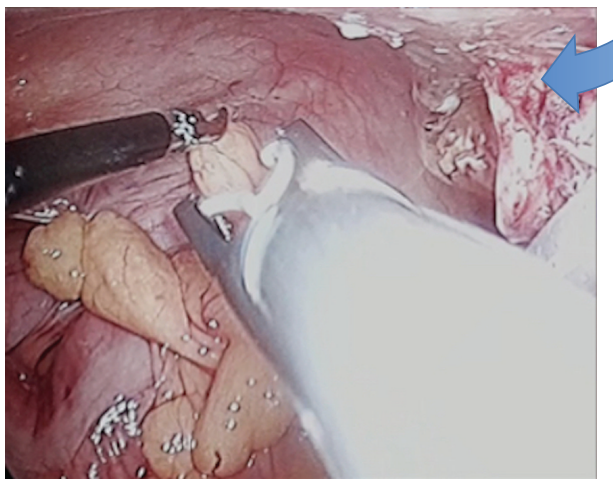

(d)

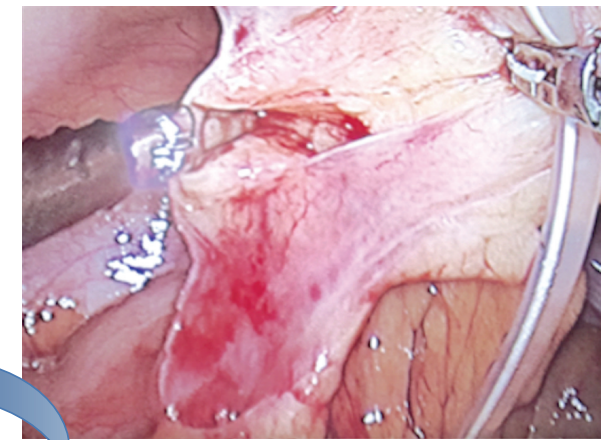

(b)

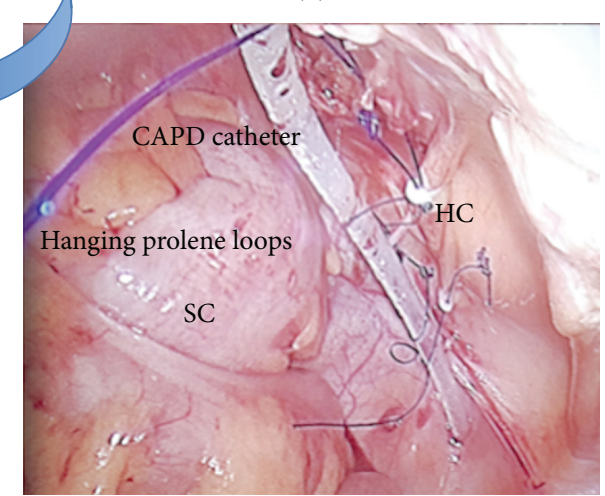

(c)

FIGURE 2: Laparoscopic view demonstrating wrapped-up CAPD catheter by appendices epiploicae of sigmoid colon (a). Dissection of the catheter from epiploicae using harmonic shear device (b). CAPD catheter hanging freely over prolene loops that are fixed to the pelvic parietal peritoneum using hemostatic clips (c). Epiplopexy using hemostatic clips (d) (AE = appendices epiploicae; CC = CAPD catheter; SC = sigmoid colon; $\mathrm{SM}=$ sigmoid mesocolon; $\mathrm{HC}=$ hemostatic clips).

two working ports (Figure 1(b)). To our surprise, the catheter was engulfed by appendices epiploicae of sigmoid colon (Figure 2). With the help of laparoscopic harmonic shear device, the catheter was completely dissected off from the appendices epiploicae. It was repositioned in the pelvis. Further, in an attempt to prevent recurrent CAPD catheter occlusion and migration, an innovative "Santosh-PGI Hanging
Loop Technique" was applied. In this, prolene 2-0 needleless suture was taken. Two knots were applied on it, one on each side. This suture was then passed into peritoneal cavity through one of the ports. A loop was made around the CAPD catheter and was fixed to the pelvic peritoneum with help of hemostatic clips. Similarly, two more loops were applied. In this way, the CAPD catheter was hanged freely 
underneath the pelvic peritoneum with the help of prolene suture. To prevent appendices epiploicae from falling back on the catheter, they were fixed to the left lower abdominal parietal peritoneum using hemostatic clips. On table catheter patency and position were confirmed. Postoperative period was uneventful. Low volume dialysis was started on 1st postoperative day. Normal volume dialysis was resumed by day 9. At 6-month follow-up his catheter is functioning well. Repeat X-ray imaging revealed its pelvic position.

\section{Discussion}

From 1997 to 2008, the number of peritoneal dialysis patients in developing countries increased by 24.9 patients per million population while in developed countries it increased by 21.8 per million population [1]. CAPD catheter malfunction is one of the major causes for discontinuation of this therapeutic modality. Incidence ranges from 12 to $73 \%$ [4]. Catheter malfunction accounts for $20 \%$ of patient transfers to hemodialysis [5]. Malfunction can result from occlusion of catheter by omental or small bowel wrapping with adhesions, fibrin clot, migration or malposition, and tunnel infections. Various series have reported omental wrapping and malposition to be the most common cause of malfunction $[2,6,7]$. Appendices epiploicae has been rarely reported as a cause of catheter occlusion [3]. CAPD catheter salvage instead of removal helps in functional rescue, thereby prolonging catheter life, decreasing patient morbidity, reducing financial burden, and allowing early institution of peritoneal dialysis, thereby decreasing dependence on intermediary hemodialysis [8]. Laparoscopic salvage of CAPD catheters has several reported advantages [8]. Laparoscopy provides a direct vision of the pathology, helps in diagnosis of other intra-abdominal pathologies, has lower incidence of postsurgical adhesions, incision related complications, postprocedure pain, thereby resulting in early ambulation, shorter hospital stay with quicker return to work. It allows early institution of peritoneal dialysis and better functional survival of catheter. Salvage procedures include laparoscopic unwrapping of omentum with omentectomy, omentopexy, [2] adhesiolysis, or laparoscopic milking of occluded catheters [9]. Laparoscopically salvaged catheters have been shown to have reasonably good period of catheter survival with a median of 163 days in some studies [7]. Initial success rates of as high as $100 \%$ have been reported [8] highlighting the usefulness of salvage procedures.

Catheter tip migration contributes to a substantial proportion of catheter malfunction. Those not corrected by conservative or stiff wire manipulation require surgical intervention. Laparoscopic salvage has definite advantages over open surgical procedures. Laparoscopic preperitoneal placement and pelvic fixation using extracorporeal knotting have been reported in an attempt to prevent catheter migration [10]. We report an innovative, easily performed technique of laparoscopic rescue of CAPD catheter malfunction due to wrapping by appendices epiploicae of sigmoid colon and catheter tip migration. In this, we first unwrapped the catheter from appendices epiploicae. The catheter was freely hanged underneath the pelvic peritoneum over prolene suture loops that were fixed to the peritoneum with hemostatic clips using laparoscopic clip applicator. The tip of the catheter was in the pelvic cavity. This technique has several advantages. Suture fixation is easy with hemostatic clips. The catheter hangs freely in the loop thereby allowing easy removal or exchange in the future. No separate incisions are required for subcutaneous suture fixation as it is done intraperitoneally. In order to prevent appendices from falling back on catheter, it was fixed to left lateral wall with hemostatic clips. This technique highlights the simplicity with which the catheter migration and occlusion can be prevented.

\section{Conclusion}

CAPD catheter malfunction is a common problem. Laparoscopic salvage of such catheters decreases patient's morbidity. The laparoscopic hanging loop technique with epiplopexy using hemostatic clips helps in effectively preventing catheter tip migration out of pelvic cavity and rewrapping of catheter by appendices epiploicae.

\section{Conflict of Interests}

The authors report no conflict of interests.

\section{References}

[1] A. K. Jain, P. Blake, P. Cordy, and A. X. Garg, "Global trends in rates of peritoneal dialysis," Journal of the American Society of Nephrology, vol. 23, no. 3, pp. 533-544, 2012.

[2] H. M. Zakaria, "Laparoscopic management of malfunctioning peritoneal dialysis catheters," Oman Medical Journal, vol. 26, no. 3, pp. 171-174, 2011.

[3] J. H. Crabtree and A. Fishman, "Laparoscopic epiplopexy of the greater omentum and epiploic appendices in the salvaging of dysfunctional peritoneal dialysis catheters," Surgical Laparoscopy, Endoscopy and Percutaneous Techniques, vol. 6, no. 3, pp. 176-180, 1996.

[4] J. Bernardini, B. Piraino, J. L. Holley, J. R. Johnston, and R. Lutes, "A randomized trial of Staphylococcus aureus prophylaxis in peritoneal dialysis patients: mupirocin calcium ointment $2 \%$ applied to the exit site versus cyclic oral rifampin," American Journal of Kidney Diseases, vol. 27, no. 5, pp. 695-700, 1996.

[5] G. Ogunc, "Minilaparoscopic extraperitoneal tunneling with omentopexy: a new technique for CAPD catheter placement," Peritoneal Dialysis International, vol. 25, no. 6, pp. 551-555, 2005.

[6] G. Kazemzadeh, M.-H. S. Modaghegh, and A. Tavassoli, "Laparoscopic correction of peritoneal catheter dysfunction," Indian Journal of Surgery, vol. 70, no. 5, pp. 227-230, 2008.

[7] S. Santarelli, M. Zeiler, R. Marinelli, T. Monteburini, A. Federico, and E. Ceraudo, "Videolaparoscopy as rescue therapy and placement of peritoneal dialysis catheters: $s$ thirty-two case single centre experience," Nephrology Dialysis Transplantation, vol. 21, no. 5, pp. 1348-1354, 2006.

[8] G. Ögünç, "Malfunctioning peritoneal dialysis catheter and accompanying surgical pathology repaired by laparoscopic surgery," Peritoneal Dialysis International, vol. 22, no. 4, pp. 454462, 2002. 
[9] C. Fourtounas, I. Maroulis, D. Karnabatidis, A. Hardalias, and J. G. Vlachojannis, "Salvage of a totally occluded peritoneal dialysis catheter by laparoscopic milking," Seminars in Dialysis, vol. 26, no. 1, pp. E8-E10, 2013.

[10] M. E. Gunes, G. Uzum, O. Koc et al., "A modified method in laparoscopic peritoneal catheter implantation: the combination of preperitoneal tunneling and pelvic fixation," ISRN Surgery, vol. 2013, Article ID 248126, 5 pages, 2013. 


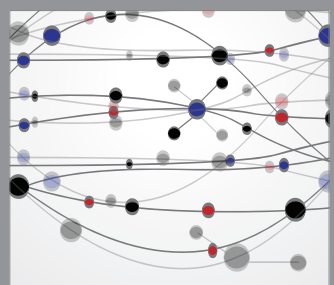

The Scientific World Journal
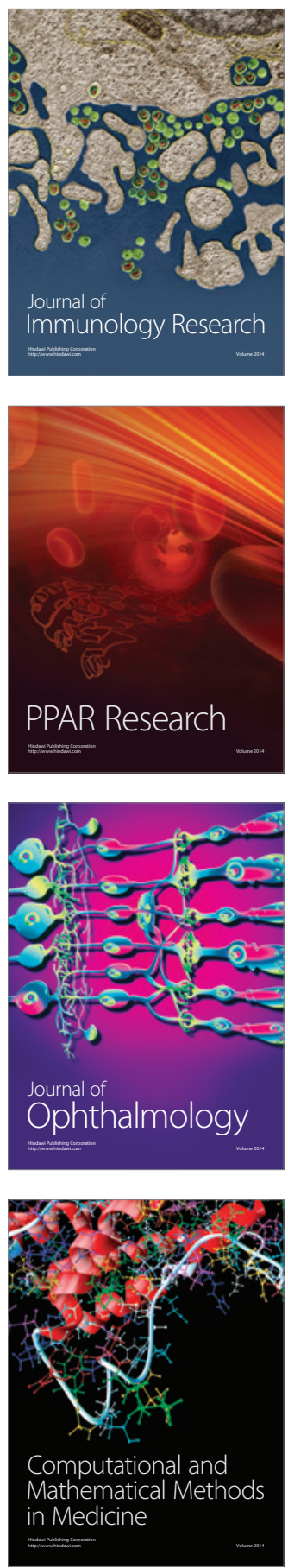

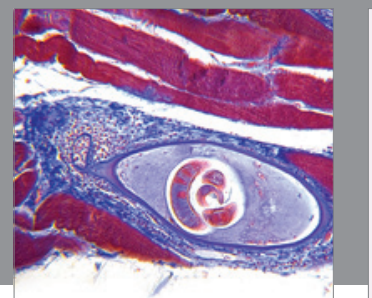

Gastroenterology

Research and Practice
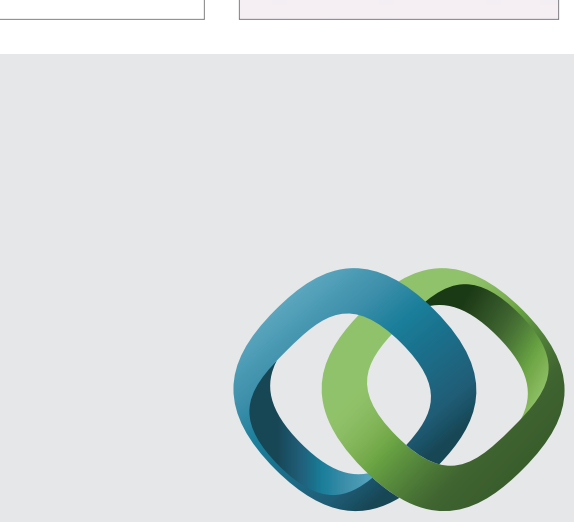

\section{Hindawi}

Submit your manuscripts at

http://www.hindawi.com
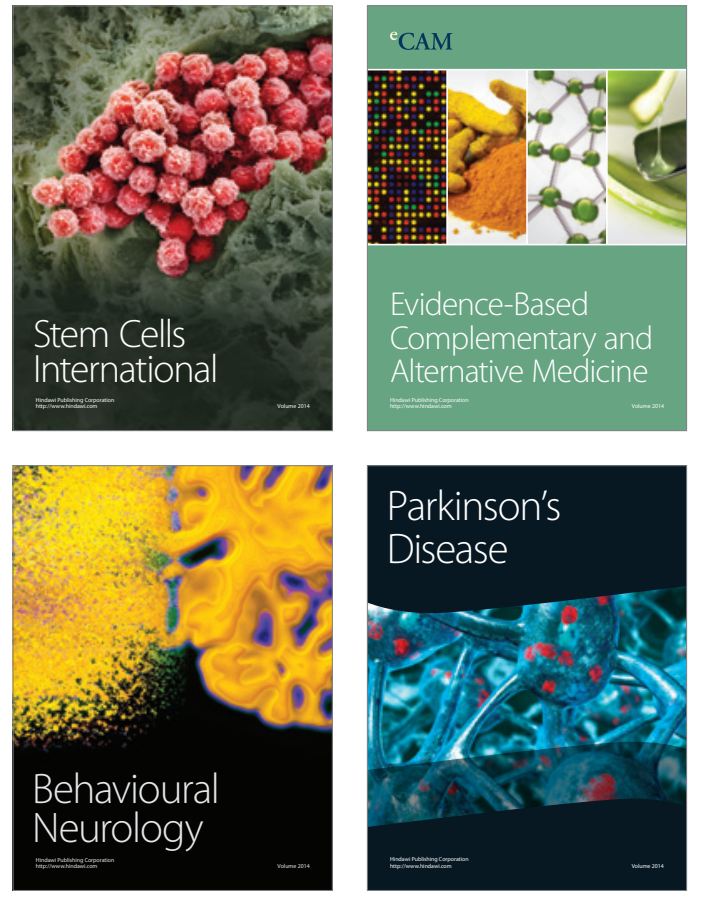
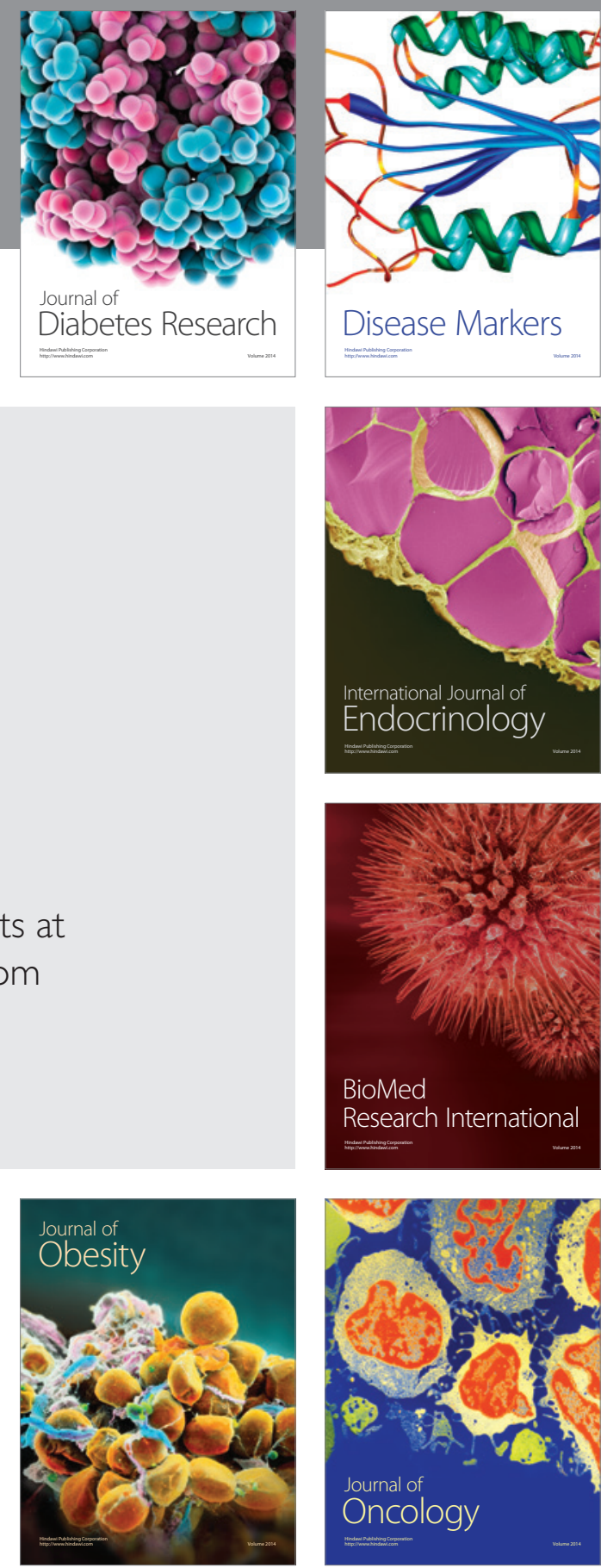

Disease Markers
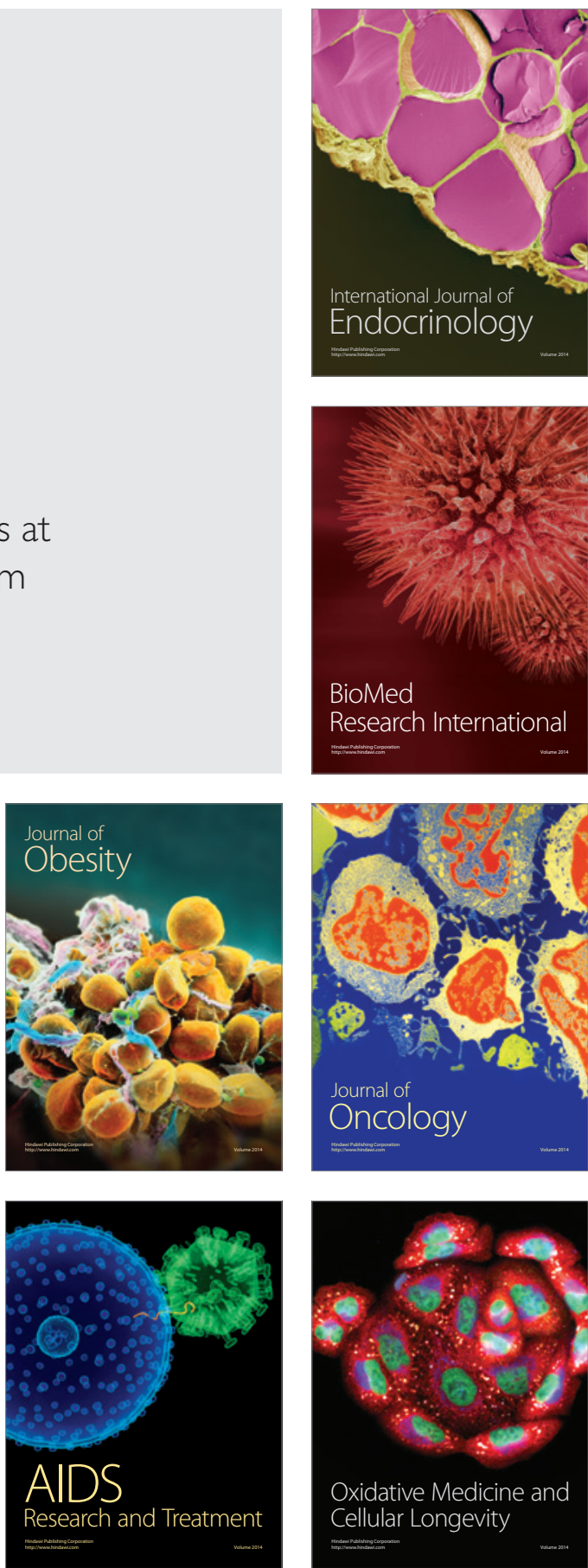